(C) 2014 IEEE. Personal use of this material is permitted. Permission from IEEE must be obtained for all other uses, in any current or future media, including reprinting/republishing this material for advertising or promotional purposes, creating new collective works, for resale or redistribution to servers or lists, or reuse of any copyrighted component of this work in other works.

This is the Author's Accepted Manuscript version of a paper published in IEEE COMMUNICATIONS MAGAZINE, by IEEE in March 2014.

The full citation of the original IEEE publication is:

Arshad, K.; Mackenzie, R.; Celentano, U.; Drozdy, A.; Leveil, S.; Mange, G.; Rico, J.; Medela, A.; Rosik, C., Resource management for QoS support in cognitive radio networks, IEEE Communications Magazine, vol.52, no.3, pp.114,120, March 2014

doi: 10.1109/MCOM.2014.6766095

The final publication is available at IEEE via http://dx.doi.org/10.1109/MCOM.2014.6766095 


\title{
Resource Management for QoS support in Cognitive Radio Networks
}

\author{
Kamran Arshad - University of Greenwich \\ Richard MacKenzie - BT Innovate \& Design \\ Ulrico Celentano - University of Oulu \\ Arpad Drozdy - Budapest University of Technology and Economics \\ Stéphanie Leveil - Thales Communications \& Security \\ Geneviève Mange - Alcatel Lucent Bell Laboratories Germany \\ Juan Rico and Arturo Medela - TST Sistemas \\ Christophe Rosik - NEC Technologies France
}

\begin{abstract}
Cognitive radio technology is a key enabler to reuse a finite, scarce and expensive resource: the radio spectrum. Guaranteeing required levels of Quality of Service (QoS) to cognitive users and ensuring necessary protection to incumbent users are the two main challenges in opportunistic spectrum access. This article identifies the main requirements and challenges for QoS support in cognitive radio networks. A framework for a two-fold cognitive manager is presented; one part managing spectrum availability on longer timescales and the other handling resource management on shorter timescales. This article gives particular focus to the functionalities of the latter cognitive manager related with resource management. Finally, we present a few key scenarios and describe how QoS can be managed with the proposed approach without disturbing the communications of incumbent users.
\end{abstract}

\section{Introduction}

Recently, there is a tremendous growth in the number of mobile broadband subscribers; posing opportunities as well as challenges to the cellular operators all over the world [1]. Further, data usage per mobile subscriber is expected to increase significantly in the coming years; this is mainly due to the attractive incentives from the service providers e.g. flat-rate tariffs in conjunction with the proliferation of large screen smart devices and video/bulky traffic. Radio spectrum is used in all wireless communications. The inefficient use of this finite resource necessitates the development of opportunistic spectrum access mechanisms in which cognitive radios can access licensed spectrum without harming the operation of the incumbent users (incumbent users may also be referred to as primary users or licensed users). These spectrum access opportunities are commonly referred to as "whitespaces" (WS).

The TV band is a prime example of a spectrum band that is currently being considered to allow for cognitive radios to access licensed spectrum. The digital switch over of TV broadcasting services is already finished in major parts of European countries and is planned to be finalized in 2012 almost all over Europe (including UK) ${ }^{1}$. In the case of TV whitespaces (TVWS), the incumbent users are the TV broadcasters and, in some countries, other devices such as wireless microphones (Programme Making and Special Events - PMSE - equipment is an example of these

\footnotetext{
1 The switch over is completed in Austria, Belgium, Czech Republic, Croatia, Denmark, Estonia, Finland, France, Germany,. Latvia, Netherlands, Norway, Slovenia, Spain, Sweden and Switzerland. It is planned to be completed during 2012 in Hungary, Ireland, Italy, Lithuania, Portugal, Slovakia and UK. Poland, Ukraine and Russia will catch up by 2013, 2014 and 2015, respectively. (Source: www.digitag.org.)
}

other devices). In the USA, the FCC has already published rules to allow for the use of TVWS [2]. It is expected that other regulators, particularly in Europe like, e.g., Ofcom started in UK [3], will soon publish their own set of rules for their own regions. The TV band exists in the UHF spectrum band which, due to its low frequency, has very good propagation characteristics. This makes TV whitespaces particularly well suited to certain scenarios such as cognitive ad-hoc networks, coverage extension in cellular bands etc. Several standards are already defined for the use of TV whitespaces such as IEEE 802.22 [4] and ECMA-392 [5], while others are due to be completed soon like IEEE 802.11 af [6] and the ETSI RRS technical report [7].

Among other challenges [1], it is important to ensure that opportunistic use of spectrum by cognitive radios does not contribute towards the potential service degradation of the incumbent users (e.g., TV broadcasting services). At the same time, it is a natural requirement for any network and service provider to provide end-to-end QoS to its own users and this should be true with cognitive radio networks. This is a challenging task as, in this case, the radio resources are shared and can be sporadic in nature.

Providing good QoS and user experience at the right price is a key to the commercial success of cognitive radio networks. For instance, investigations conducted for collaborative cognitive radio resource management algorithms in [8] were based on QoS metrics together with network statistics in order to show the benefits expected for the overall system performance.

To cope with the peculiar challenges described in the following section (Figure 1), the parts of the proposed two-fold cognitive manager described later in this article (Figure 3) address the relative duties. In this article, we describe how a cognitive radio network may be controlled by the cognitive manager (CM) for spectrum management (CM-SM) and resource management (CM-RM) with a particular focus on the cognitive resource management part (Figure 4) which handles all the operations related to resource control and usage. The CM-RM also plays an important role in incumbent protection which will be described in detail.

The rest of this article is organized as follows. we first discuss the QoS requirements and major challenges in cognitive radio networks. Then, we describe our proposed approach to tackle challenges identified in the previous section. Finally, we detail the applications of our solution approach in a few selected scenarios and then conclude the article. 


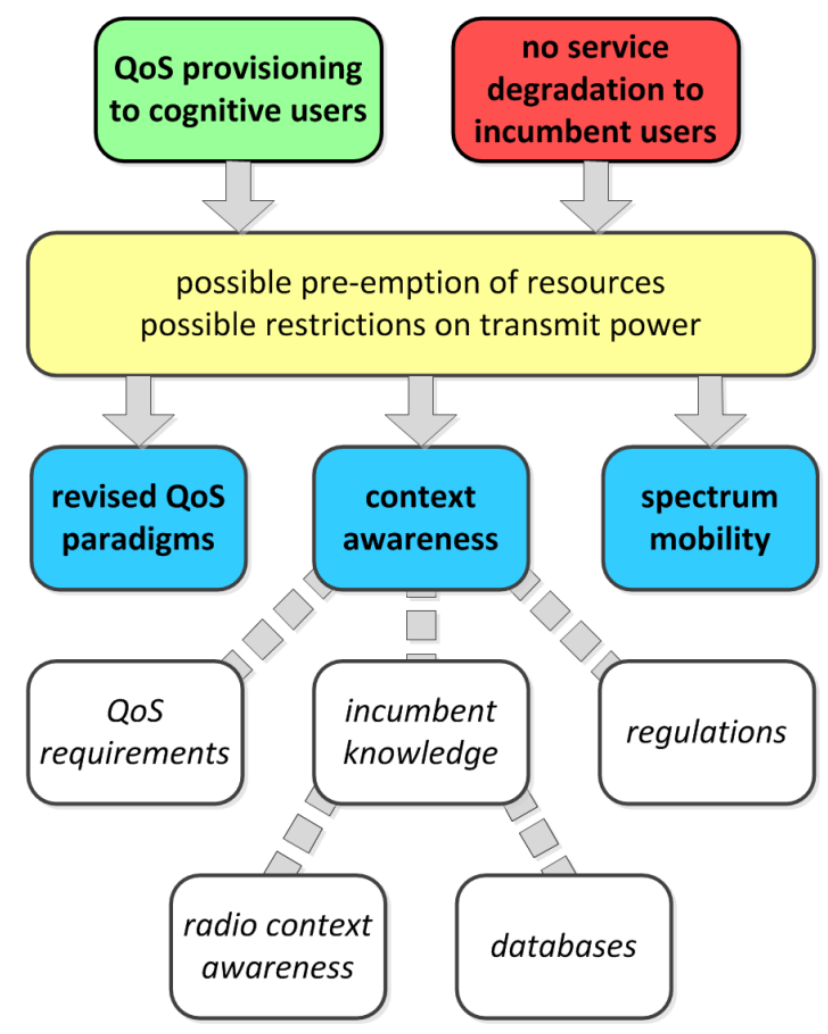

Figure 1 Constraints about QoS provision and incumbent protection, and high-level requirements for cognitive radio networks

\section{QoS Challenges and Requirements in Cognitive Radio Networks}

The deployment of cognitive radio technology raises new challenges to manage the radio resources while the protection of the incumbents must be addressed as a matter of priority. Incumbent users should not be faced with service degradation due to interference caused by the presence of cognitive radios, and this puts limitations on the provision of cognitive services. Available whitespaces may not necessarily offer the bandwidth needed to provide a given service for the cognitive users and the protection of incumbent users from interference restricts the service level offered so that the required QoS has to be optimized.

Figure 1 summarizes the derivation from the constraints of challenges and high-level requirements as far as QoS is concerned. A cognitive radio network which makes use of whitespaces faces the extra challenge that its users are opportunistic users, and there are incumbent applications which are non-cognitive and may unexpectedly take control of spectral resources. When an incumbent starts using (or it is close to do so) any particular channels the use of these channels by cognitive radios will be terminated abruptly, imposing a very serious challenge for providing QoS guarantees: the cognitive user must vacate the resource and the service needs to be promptly handed over to another available spectrum portion (spectrum mobility).

Some key requirements from [9] accounting for both the QoS and resource management for cognitive radios and the protection of incumbents are presented in Table 1. These requirements imply the need to develop advanced cognitive mechanisms using knowledge of the relevant regulatory constraints, of the available resources and of the QoS needs of the cognitive radios. In particular, this includes the knowledge about the appearance of an incumbent user which can be a priori or a posteriori [10], i.e., achieved by using geo-location/geographical databases or spectrum sensing, respectively. These two methods of information gathering (spectrum environment awareness) can be used in an integrated way, to exploit the benefit of each (as discussed in the $10^{\text {th }}$ meeting of the CEPT SE43 working group in July 2011 [11]), or alternatively, depending on the regulatory rules.

\begin{tabular}{|c|c|}
\hline \multirow{5}{*}{$\begin{array}{l}\text { QoS for } \\
\text { cognitive } \\
\text { users }\end{array}$} & $\begin{array}{l}\text { The required level of QoS should be maintained in } \\
\text { presence of variations in the available spectrum } \\
\text { resources. }\end{array}$ \\
\hline & $\begin{array}{l}\text { The quality level of radio environment awareness shall } \\
\text { depend on the QoS requirements. }\end{array}$ \\
\hline & $\begin{array}{l}\text { The reserve channels for use when an incumbent is } \\
\text { detected shall be identified according to QoS needs. }\end{array}$ \\
\hline & $\begin{array}{l}\text { The admission control shall depend on the QoS } \\
\text { requirements and on the channel capacity of the } \\
\text { available resources. }\end{array}$ \\
\hline & $\begin{array}{l}\text { Efficient allocation of the opportunistic resources shall } \\
\text { fulfill the QoS needs inside given bounds specific to the } \\
\text { scenario. }\end{array}$ \\
\hline \multirow{4}{*}{$\begin{array}{l}\text { Incumbent } \\
\text { protection }\end{array}$} & $\begin{array}{l}\text { Cognitive networks shall have the capability to evict } \\
\text { users upon resource shortage, for example due to } \\
\text { incumbent user apparition. }\end{array}$ \\
\hline & $\begin{array}{l}\text { Cognitive networks shall schedule quiet periods for } \\
\text { spectrum sensing purpose, when required, without } \\
\text { degrading the QoS of the cognitive users. }\end{array}$ \\
\hline & $\begin{array}{l}\text { Cognitive networks shall vacate their operating channel } \\
\text { upon appearance of an incumbent user, or use a transmit } \\
\text { power allowing simultaneous operations. }\end{array}$ \\
\hline & $\begin{array}{l}\text { The transmission of information related to the presence } \\
\text { of an incumbent shall have priority over data } \\
\text { transmission. }\end{array}$ \\
\hline
\end{tabular}

Table 1 Key requirements for QoS and resource management, and for incumbent protection in cognitive radio networks

\section{How Can QoS Be Controlled?}

To fully appreciate the challenge of providing end-to-end QoS in a cognitive radio network, it is important to be aware of the wide range of actors involved. End users, not worried about how services are provided, request an acceptable QoS level. Content providers offer applications with different delivery requirements through service providers, who acquire connectivity and capacity from network operators. A spectrum database owner provides extra spectrum environment awareness information to cognitive radios. The amount of available resources has a huge impact on the levels of QoS that can be provided. Service Level Agreements (SLA) set the service levels that will be supplied and fix the penalties when the parameters agreed are not fulfilled. Figure 2 depicts the relationship among the actors and their agreements in the end-toend QoS chain. 


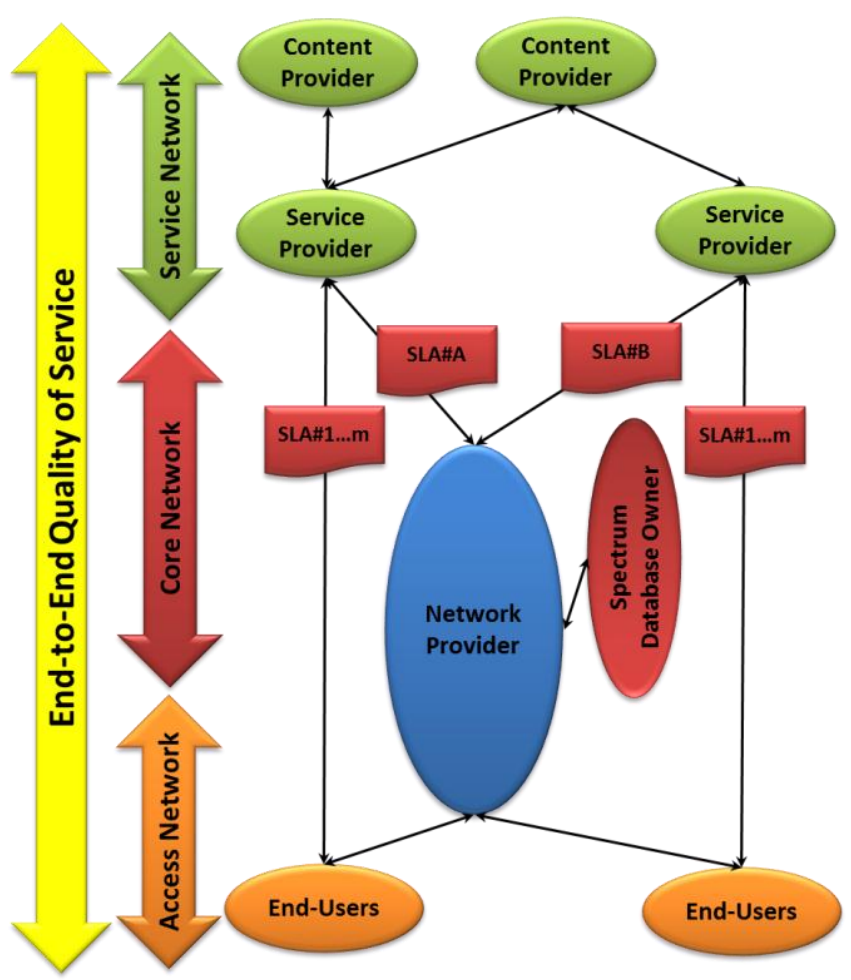

Figure 2 Configuration including multiple service providers

The key novelty introduced in this architecture, designed for optimizing QoS management mechanisms in cognitive radio systems, is the presence of a two-fold cognitive manager. This QoS management proposal acts at various levels; on the one hand, the CM-SM identifies the available spectrum and builds a spectrum portfolio that operates on a larger time scale. On the other hand, the CM-RM makes maximum use of the existing resources and divides them efficiently among users, operating on a shorter time scale. Use of the spectrum will become more efficient if local decisions focus on what spectrum to occupy for a particular service at a particular time. If a mix of distributed triggers (e.g. from the user terminals) and centralised ones (e.g. from the network) can be used in a cognitive decision making process, cost savings will be achieved through massive reuse of spectrum resources.

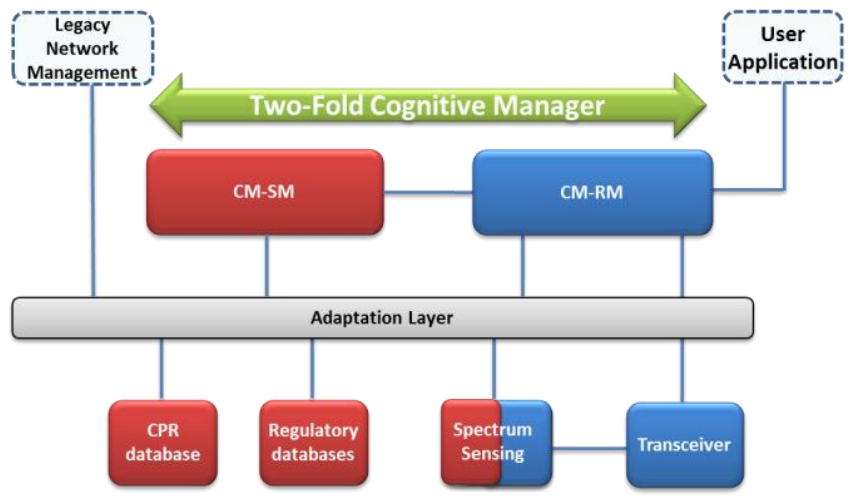

Figure 3 Overall System Architecture

Figure 3 depicts the proposed architecture. It contains, besides the CM-RM and CM-SM, two main databases storing information about spectrum usage and regulatory constraints, a block for spectrum sensing, a transceiver focused on physical layer interactions and an adaptation layer in charge of facilitating information exchange among the different blocks.
In order to provide reliable links, the CM-SM attempts to keep as much usable spectrum identified as possible. The CM-RM will then distribute this broad range of resources among cognitive radios, and maintain open channels on as many frequencies as possible, some only as backup. The CM-RM keeps a list of operating channels and backup channels allowing users to carry on with their transmissions without a temporary halt, in the case that some channels become unavailable. However, the CM-RM has to cope with any decreases in available bandwidth. Due to this, conventional radio networks handovers may decrease QoS; however, our proposal performs a completely seamless handover mechanism exploiting diverse resources in the most efficient manner.

There are many options, based on the chosen network topology, for how the functional entities of this proposed architecture can be mapped to system elements [12] [13]. For centralised topologies, the CM-SM functionality will be placed in access points/base stations and also, if present in the topology, partly in the core network. In distributed topologies, the CM-SM also has to be present in user equipment so that each CM-RM can receive necessary portfolio information. As described in the following, in all kinds of topologies the CM-RM will be present in controllers (e.g. access points/base stations) and user equipment so that resources can be exploited in both the downlink and uplink.

To achieve its goals, the CM-RM is organized into two functional groups in charge of the respective duties at the terminating and networking domains [12] and correspondingly involved in resource usage (RU) and resource control (RC) (see Figure 4).

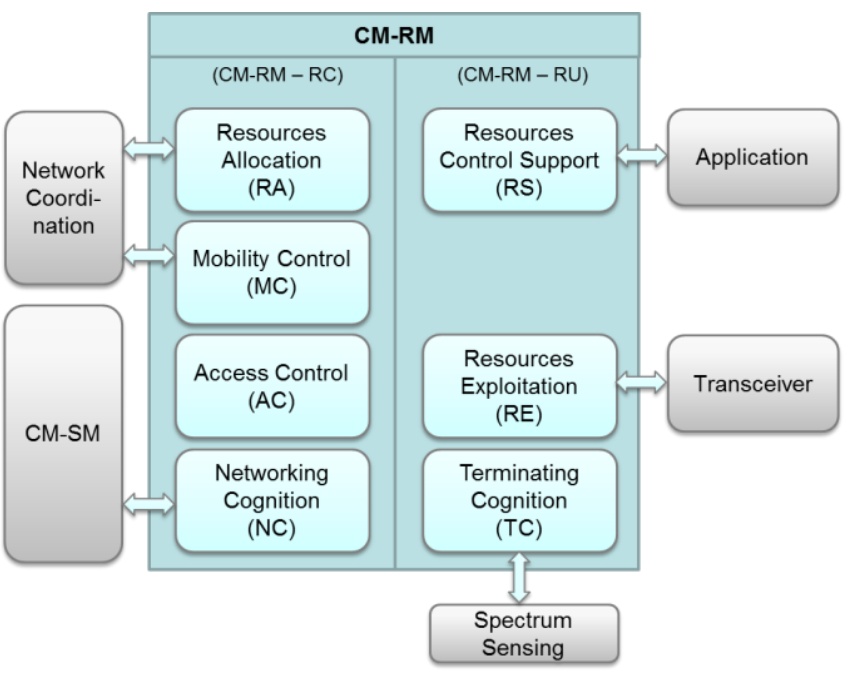

Figure 4 CM-RM overview

There are three functional entities within RU; the first of these is the terminating domain cognition (TC). This controls the gathering of information on the environment in the terminating domain. This information can aid in mobility support and also for the configuration and collecting of sensing measurements. The second entity within RU is resource exploitation (RE). This provides the data service to upper layers and optimizes the use of lower layers. The lower layer optimizations include mapping logical channels to physical channels and configuring the transceiver. The final entity in RU is resource control support (RS), which is in charge of supporting at terminating domain the resource management done at the networking domain. This 
includes such things as the configuration of the transceiver through the RE and executing decisions made by the TC.

The first of the four functional entities within the $R C$ is resource allocation (RA) which assigns resources based on current QoS constraints and information from the CM-SM. This also includes the reallocation of resources when an incumbent appears. The access control (AC) entity will gather context information to make admission decisions for service requests. This will also be involved in handover decisions. The networking domain cognition (NC) entity gathers environmental information relevant to the networking domain. This includes spectrum portfolios from the CM-SM and collecting measurement of performance and sensing from active and backup channels (sensing only in backup channels). Finally, the mobility control (MC) entity provides mobility support and execution of mobility management. This is for both spectrum mobility and physical mobility.

The mapping of RU and RC entity groups to network devices in done accordingly to the presence of centralised or distributed resource control [12]. With centralised resource control, as in an ad hoc network with star topology or a cellular access network, the $\mathrm{RC}$ is located at the gateway device or the base station, respectively. With distributed resource control, the RC is present in any peer device. The RU is needed in any device having a radio interface and is therefore present in user equipments, base station and peer devices in the above examples. Those examples are further illustrated and discussed in the following section.

\section{QoS Management in Selected Scenarios}

An important functionality of the CM-RM is to make sure that incumbents and cognitive radios QoS are satisfied simultaneously during the opportunistic spectrum access. In order to demonstrate the applicability of mechanisms discussed in this article, in this section we discuss a few applications of cognitive networks and illustrate how QoS is maintained in these scenarios.

\section{Cognitive Ad-hoc Scenario}

In this scenario, cognitive radios at a particular location (e.g. home, office, shopping centre etc.) setup an ad-hoc network to efficiently communicate with each other. Such an ad-hoc network may be created to fulfil simple tasks like file sharing to more complex tasks like providing access to Internet (e.g. relaying other terminals in the ad-hoc network). Cognitive ad-hoc networks are typically limited in space and time and opportunistically access whitespaces for communications.

Depending on the scenario and application, a cognitive ad-hoc network can be created for example with a star or mesh topology. In star topology, there is a master node that is responsible for all resource management related functions. In this case, the CM-SM block within the master node establishes a connection to the spectrum databases in order to get environment awareness as well as comply with regulatory needs. In cases when the regulatory regime does not require the use of a database, the incumbent protection constraints translate into stricter requirements on spectrum sensing (for environment cognition). The CM-RM of the master node manages resources within the cognitive ad-hoc network locally.

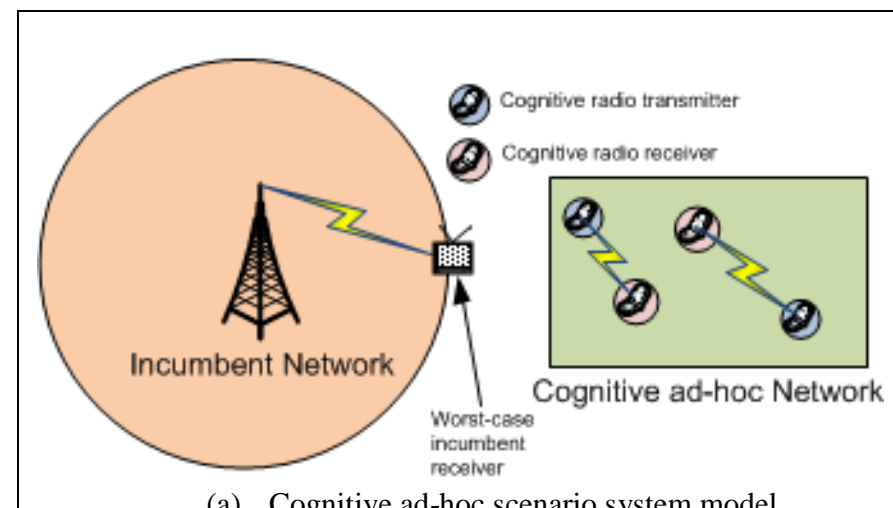

(a) Cognitive ad-hoc scenario system model

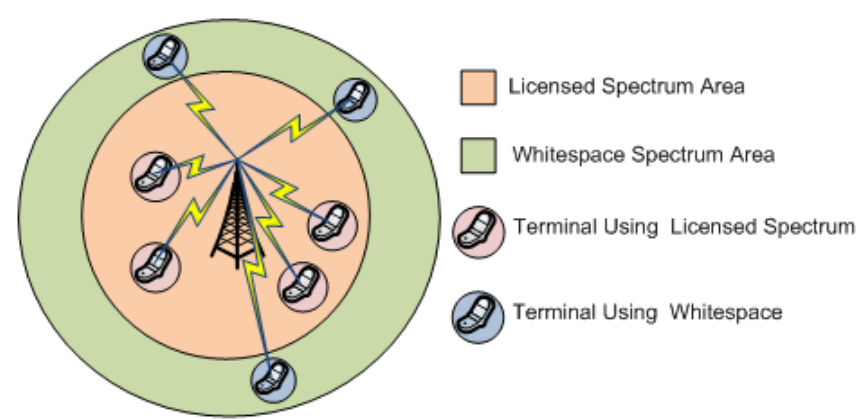

(b) Cellular extension scenario enhancing coverage in unlicensed frequency band(s)

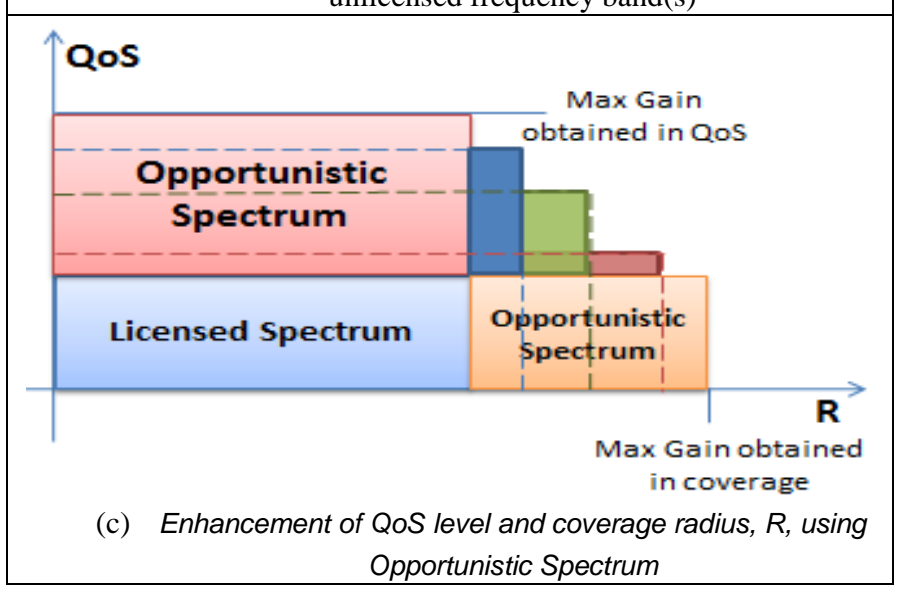

Figure 5 Examples of QoS Management for Cognitive Radio Systems

In a cognitive ad-hoc network with a mesh topology, there is no master node and hence distributed operation of cognitive radios is required. In this case, cognitive radios are autonomous ${ }^{2}$ and to fulfil tight QoS constraints for cognitive radios as well as incumbent protection is a challenging task. As an example, a system model for sharing TV whitespaces by a cognitive ad-hoc network is shown in Figure 5a.

To demonstrate the efficacy of the concepts presented in this article, we assume the worst-case location of a TV receiver (incumbent) by arguing that if the worst-case user is protected then all the incumbent receivers within the coverage area of the incumbent transmitter are also protected. In this setting, the CM$\mathrm{RM}$ of each cognitive radio fetches portfolio information from the CM-SM and initiates a sensing phase. All cognitive users perform

\footnotetext{
${ }^{2}$ The ad hoc network considered here is autonomous in that it does not rely on external entities for its control. However, in compliance with regulations, it may need to exchange information with external entities through a CM-SM, even if this connection is not continuously needed.
} 
spectrum sensing to estimate their distance from the worst case incumbent receiver. Based on the estimated distance from the incumbent receiver (for details refer to [14]), cognitive users initiate Distributed Power Control (DPC) algorithms to simultaneously guarantee QoS requirements for their respective receivers, but also to avoid harmful interference to the incumbent. QoS requirements for incumbents and cognitive radios can be stated in terms of tolerable interference at incumbents and signal to noise-plus-interference thresholds at cognitive radios [14]. Simulation results and details of distributed algorithms are detailed in [14] demonstrating that the QoS of incumbent users is always protected.

\section{Cellular Extension in Whitespace}

This scenario aims at cellular networks utilizing whitespace spectrum in addition to their own licensed spectrum, thus gaining additional bandwidths to benefit the user. This is shown in Figure $5 b$.

The use of the WS spectrum must be shared among the different mobile operators. The information provided by the CM$\mathrm{SM}$ will be exploited in the most effective way by the CM-RM. The main advantages derived from the use of the whitespaces lie on two main aspects (Figure 5c):

- Enhanced coverage area. Addressing long range requirements by using low frequency whitespaces such as TV whitespaces.

- Enhanced capacity. Fulfilling short range requirements.

Depending on the QoS required by the different services offered to the users, the configuration of each connection will be modified according to the needs and availability. This process carried out by both cognitive managers takes into consideration not only the availability of spectrum but also the services contracted by the different users.

Thus, the CM-RM may take the advantages of both licensed and unlicensed spectrum characteristics to optimize the utilization of available radio resources. As it is not possible to entirely guarantee the QoS by an exclusive access to the whitespace spectrum, the CM-RM may, for example, assign these opportunistic resources to low priority services while reserving the licenced band to high priority services, such as, emergency communications. Another example is where cognitive managers, deployed in networks supporting spectrum aggregation, may use the available radio resources in a more flexible way, mapping signalling logical channels onto the licensed band (in order to guarantee user connection, even upon incumbent apparition) and mapping user data logical channels onto the whitespace band. In this context, CM-RM implements key enabling decision-making mechanisms for supporting user QoS while protecting incumbent operations.

One illustration of these protection measures is the spectrum mobility which consists in forcing a cognitive user to handoff from its initial cell to a neighbour or co-located cell operating in a channel other than the one pre-empted by the incumbent. This contributes to release the relevant radio resources in the impacted cell and to limit the QoS degradation of the served connections. In parallel, the cognitive manager adapts its admission control decisions for supporting this unexpected increase of handover demands, in order to prevent degrading the dropping probability in the neighbour cells as well as the blocking probability in the impacted cell.
The features of this scenario are beneficial for the operators, obtaining extra operational bandwidth resulting from load balancing, improving link quality, and offering more flexible services, thus enhancing user experience.

\section{Conclusions}

Cognitive radio networks, accessing licensed spectrum on an opportunistic basis, can vastly improve the efficient use of the radio spectrum. This benefit can help to support the rapidly growing demand for high-rate and multimedia-rich traffic in wireless networks. This paper explains that for this benefit to be realised, a cognitive radio network must employ advanced QoS mechanisms for its opportunistic users whilst ensuring, at all times, that incumbent users of spectrum are protected.

This paper highlights some of the main challenges facing cognitive radio networks and describes a two-fold cognitive manager that can allow for these challenges to be resolved, ensuring QoS and incumbent protection at the same time. Particular attention is paid to the resource management aspects of the cognitive manager which provides resources to opportunistic users over short time scales. Selected scenarios have been described to demonstrate how this cognitive manager can provide various QoS mechanisms. These scenarios, cognitive ad-hoc networks and cellular extension in whitespaces, act as just a small flavour of the vast range of potential scenarios for cognitive radios that could thrive in the future.

\section{References}

[1] J. Peha, "Approaches to spectrum sharing," IEEE Communications Magazine, vol.43, no.2, pp.10-12, Feb. 2005

[2] FCC, "Second memorandum opinion and order in the matter of unlicensed operation in the TV broadcast bands, additional spectrum for unlicensed devices Below $900 \mathrm{MHz}$ in the $3 \mathrm{GHz}$ Band", Sept , 2010, Document 10-174.

[3] Ofcom, Digital dividend: consultation on license-excepting cognitive devices using interleaved spectrum, May 2009

[4] IEEE 802.22 Cognitive Wireless Regional Area Networks (RAN) for Operation in TV Bands, published July 2011

[5] ECMA-392 MAC and PHY for operation in TV White Space, published Dec 2009

[6] IEEE 802.11af/draft 1.03 Amendment 4: TV white spaces operation, Sept 2011

[7] ETSI RRS TR102 907 Use cases for operation in white space frequency bands v. 0.1.7 Sept 2011

[8] End-to-End Efficiency (E3) Deliverable D3.3 "Simulation based recommendations for dynamic spectrum assignment and selfmanagement", , July 2009

[9] G. Mange, C. Rosik, S. Leveil, U. Celentano, O. Durowoju, K. Arshad, "Cognitive resource management for QoS support in mobile opportunistic communications", Proc. Future Network \& Mobile Summit (FuNeMS), Warszaw, Poland, 15-17 Jun 2011.

[10] P. H. Lehne, D. Noguet, R. Datta, U. Celentano, G. Fettweis, "Requirements for a CR-system - challenges compared to conventional wireless technology", Proc. Wireless Innovation Forum European Conference on Communications Technologies and Software Defined Radio (WInnComm), Jun 2011

[11] Minutes of the $10^{\text {th }}$ meeting of ECC CEPT SE43 working group, Bologna, 5-7 July 2011 http://www.cept.org/Documents/se43/661/SE43_11_40_Minutes_of_the_10th_meeting

[12] U. Celentano, B. Bochow, C. Lange, F. Noack, J. Herrero, B. Cendón, O. Grøndalen, V. Mérat, C. Rosik, "Flexible architecture for spectrum and resource management in the whitespace", Proc, Int. Symp. Wireless Personal Multimedia Commun. (WPMC), Oct 2011

[13] QoSMOS Deliverable D2.3, "System specification and evaluation criteria", Nov 2011

[14] O. Durowoju, K. Arshad, and K. Moessner, "Distributed power control for cognitive radios with primary protection via spectrum sensing," IEEE Vehicular Technology Conference Fall, pp.1-5, 6-9 Sept. 2010. 


\section{Acknowledgements}

The research leading to these results was derived from the European Community's Seventh Framework Programme (FP7) under Grant Agreement number 248454 (QoSMOS).

Kamran ARSHAD received his Bachelor of Engineering and Master of Science degrees in Electrical Engineering with distinction. He completed his PhD in computing science in 2007 and currently associated with University of Greenwich. He is an author of more than 50 technical publications in peer reviewed Journals and International Conferences and holds 3 best paper awards. Dr Arshad research interests include spectrum sensing, radio resource management for $3 G / 4 G$ systems and channel modeling. He contributed in several EU projects including E3, ORACLE and QoSMOS.

Richard MACKENZIE received his MEng degree in Electronic Engineering from the University of York in 2005 and his PhD in Electronic \& Electrical Engineering from the University of Leeds in 2010. He has worked at BT Innovate \& Design since 2009 as a researcher in wireless communications. His work covers many areas of cognitive radio with a particular interest in the use of TV whitespaces. His work includes mathematical analysis, system simulations and test-bed implementations.

Ulrico CELENTANO received his Dott.Ing. degrees in Electronic Engineering from the University of Florence, Italy. He currently is with the Centre for Wireless Communications, Department of Communications Engineering, University of Oulu, Finland. His present research interests include reconfigurable concurrent wireless networks, dependable wireless ecosystems, communication systems modeling and optimization.

Arpad DROZDY received his master degree in Electric Engineering in 2007 from the Budapest University of technology and Economics where he is currently a researcher at the Department of Broadband Infocommunications and Electromagnetic Theory.

Stéphanie LEVEIL graduated from ENSEIRB in 1995, and received the Ph.D. degree in signal processing from Bordeaux I University in 1998. During a post-doctoral year at the CEA, she applied signal processing techniques to fMRI. Then she joined Motorola Labs to work on the design of multiple-antenna and multi-user approaches for wireless communications. As a senior research engineer, she contributed to several EU collaborative projects and to IEEE 802.11 n standardization body. In 2010, she joined Thales Communications. Her current research interests include cognitive radio and ad-hoc networks. She authored or coauthored more than 30 papers and 16 patents.

Geneviève MANGE is a senior $R \& D$ engineer in the Wireless Research domain at Alcatel-Lucent Bell Laboratories in Stuttgart, Germany. She received her diploma degree from CPE Lyon, France with a specialization in Signal Theory and Processing. She has been involved in various research and development projects on mobile radio communication and in 3rd Generation Partnership Project (3GPP) standardization. She contributed to a number of European Union funded research projects including Wireless World Initiative New Radio (WINNER) I and II and End-to-End Efficiency (E3). She is currently involved in the FP7 project Quality of Service and MObility driven cognitive radio Systems (QoSMOS) focusing on cognitive QoS management in opportunistic networks.

Juan RICO received a degree in Telecommunications Engineering in 2006 and a Master degree in Information Technology and
Communication in Mobile Networks in 2009 from the University of Cantabria Spain. He has been working as Research Fellow at Network Planning and Mobile Communications Laboratory in University of Cantabria from 2006 to 2010, participating in many different $R \& D$ projects (European FP and Celtic and Spanish CENIT programs). Currently, he is a Senior Researcher working at TST R\&D Division with strong background on network management protocols, handover techniques, M2M systems, and heterogeneous access networks.

Arturo MEDELA holds a degree in Telecommunications Engineering received in 2007 from University of Cantabria, Spain. He is an R\&D Senior Engineer working for TST mainly in the field of wireless communications systems in both national and European projects. His activities cover network protocols development and also the architecture and design of cellular networks. He is currently involved in the implementation of a system to improve energy efficiency in cellular networks and co-leading the design and implementation of QoSMOS Adaptation Layer.

Christophe ROSIK holds an Engineering Degree received in 1998 from Polytech'Lille, France. He is an R\&D Senior Engineer working for NEC Technologies in the field of wireless communication systems. His activities cover architecture \& design of cellular networks and mobile phone handsets. He is involved in designing architecture based on LTE technology and is charged to handle technical studies on communication systems, such as hybrid terrestrial/satellite networks. In the area of European project, Christophe has contributed to the architecture of E3 system, focusing on the femtocell-based applications and is currently leading QoSMOS system architecture studies. 